H I G H L I G H T S

SIGNALLING

\section{A little fat ... could go a long way}

Scientists working on Wnt proteins and those interested in stem cells now have good cause to celebrate. Active, pure Wnt has at last been isolated! A joint venture by the groups of Roel Nusse, Irving Weissman and Tannishtha Reya has shown that Wnts are palmitoylated and that Wnt signalling can induce self-renewal of haematopoietic stem cells (HSCs).

Wnt molecules regulate many aspects of development, such as stemcell proliferation, but studying these processes ex vivo has been hampered by the inability to purify significant amounts of soluble, active Wnts. So Karl Willert and colleagues, from Nusse's lab, subjected medium from Wnt-expressing (and secreting) cells to chromatography in the presence of a detergent, size-exclusion chromatography, and then cation exchange to obtain fractions of $>95 \%$ pure Wnt (in this case Wnt3a). Importantly, this Wnt3a could still stabilize cytosolic $\beta$-catenin, a known target of Wnt.

That detergent was required throughout purification implied that Wnts were hydrophobic, and metabolic labelling indicated that $\mathrm{Wnt} 3 \mathrm{a}$ was palmitoylated. Further analysis showed that the most amino-terminally conserved cysteine residue of Wnt-family members (C77 in Wnt3a) was the target. Mutation of this residue, although not affecting the level of secreted protein, conferred a loss of hydrophobicity and rendered the protein inactive in $\beta$-catenin stabilization assays. Consistent with the importance of this residue, natural loss-of-function alleles of Drosophila $w g$ and of C. elegans egl-20 (which are homologues of Wnt) occur, both of which affect this conserved cysteine.

Weissman, Nusse and Reya then showed that purified Wnt3a induces proliferation of HSCs, which give rise to all lineages of the blood. Unpurified Wnt3a, by contrast, induced differentiation, emphasizing the importance of being able to purify Wnt. Wnt3atreated HSCs were then transplanted into lethally irradiated mice and, 6 weeks later, $100 \%$ of the mice contained donor-derived cells. This frequency of reconsitution indicated that self-renewal had occurred in vitro.

In a second paper, Weissman, Reya and colleagues showed the importance of the Wnt signalling pathway as a whole in HSC homeostasis in vitro and in vivo. In long-term experiments, expressing constitutively active $\beta$-catenin maintained HSCs in an immature state while allowing them to proliferate. Transplantation analysis again indicated that the expanded HSCs were functional. Using a reporter assay, the authors verified that HSCs in their normal microenvironment respond to endogenous, canonical Wnt signalling. Wnt signalling is also required for normal HSC growth; expression of a soluble form of the Frizzled cysteine-rich domain, or ectopic expression of Axin (which increases $\beta$-catenin degradation) both of which inhibit Wnt signalling - inhibited HSC growth. In vivo, transplantation of axin-expressing HSCs into irradiated mice inhibited reconstitution of their haematopoietic system. The final finding was that HoxB4 and Notch1, which were previously implicated in HSC self-renewal, were upregulated in response to Wnt signalling.

Wnt-signalling-pathway components have been implicated in regulating the proliferation of progenitor cells of the skin, gut and brain. The finding that soluble Wnt3a can induce proliferation of HSCs adds to the potential of using Wnt signalling as “.... general cue for self-renewal in stem and/or progenitor cells from diverse tissues". Importantly, Wnt signalling might facilitate expansion of a patient's or allogeneic donor's HSCs as a potential future transplantation source. Finally, the authors propose that Wnt/ $\beta$-catenin signalling might also be an important regulator of cancer stem-cell self-renewal.

\section{2) References and links} ORIGINAL RESEARCH PAPERS Willert, K. et al. Wnt proteins are lipid-modified and can act as stem cell growth factors. Nature 2003 Apr 27 (DOl: 10.1038/nature01593) |Reya, T. et al. A role for Wnt signalling in self-renewal of haematopoietic stem cells. Nature 2003 Apr 27 (DOI: 10.1038/nature01611)

\section{STRUCTURE WATCH}

\section{Sensitive to change}

Voltage-dependent $\mathrm{K}^{+}$channels open and conduct ions in response to changes in membrane voltage. But, how do these channels 'sense' membrane voltage and how do they open in response to a particular voltage? Work by MacKinnon and colleagues now published in Nature provides new insights. They determined the 3.2- $\AA$-resolution crystal structure of full-length KvAP - a voltage-dependent $\mathrm{K}^{+}$channel from Aeropyrum pernix — and the 1.9- $\AA$-resolution crystal structure of the isolated voltage-sensor domain, both in complex with monoclonal Fab fragments.

The KvAP channel is a tetramer, and its subunits are composed of six hydrophobic segments (S1-S6). S5 and S6 of each subunit line the pore and determine ion selectivity, whereas S1-S4 form the voltage sensors. Although KvAP has a canonical $\mathrm{K}^{+}$pore, the authors saw that the surrounding voltage sensors have an unexpected structure. S1 and S2 form a layer of helices outside S5, and $\mathrm{S} 3$ and $\mathrm{S} 4$ are located on the pore's outer perimeter.

Surprisingly, the $\mathrm{S} 3$ segment is actually composed of two helices (S3a and S3b), and the S3b-S4 helix-turn-helix structures form so-called voltage-sensor paddles. The paddles are mainly hydrophobic, except for $\mathrm{S} 4$ arginine residues, and they can move with respect to the pore. A 'flip' movement of these paddles in response to membrane voltage changes would be linked to movements of the pore through the S4-S5 linker. And, in another study published in the same issue of Nature, MacKinnon and co-workers describe how far these paddles move when the pore opens in response to membrane depolarization.

REFERENCE Jiang, Y. et al. X-ray structure of a voltage-dependent $\mathrm{K}^{+}$channel. Nature 423, 33-41 (2003)

\section{High fidelity}

Aminoacyl-tRNA synthetases attach amino acids to their cognate tRNA, and the precision of this reaction is crucial for the fidelity of protein synthesis. To enhance fidelity, some of these synthetases use hydrolysis to 'edit' incorrectly activated amino acids (aminoacyl-adenylates; 'pre-transfer editing') or mischarged tRNAs (aminoacyl-tRNAs; 'post-transfer editing'). And, because these substrates are distinct, it was thought that there must be separate amino-acid binding sites for these editing reactions. However, in Molecular Cell, Martinis, Cusack and colleagues now show that these binding sites essentially overlap.

The authors determined the crystal structure of leucyl-tRNA synthetase bound to analogues of pre- and post-transfer editing substrates. They found that the synthetase's editing active site binds these substrates so that the common groups - the adenine and amino-acid moiety - are bound in the same specificity pockets and are recognized in the same way. A conserved aspartic acid in the synthetase interacts with the $\alpha$-amino group of the non-cognate amino acid in both substrates, and this aspartic acid is essential for editing. It proofreads amino-acid side chains in the discrimination pocket, and also positions substrates for hydrolysis by water (the authors propose that the editing-site residues have no direct catalytic effect). This work shows "...the economy by which a single active site accommodates two distinct substrates in a proofreading process critical to the fidelity of protein synthesis". REFERENCE Lincecum, T. L. et al. Structural and mechanistic basis of pre- and posttransfer editing by leucyl-tRNA synthetase. Mol. Cell 11, 951-963 (2003) 